\title{
Fold Conservation and Proteolysis in Zebrafish IRBP Structure: Clues to Possible Enzymatic Function?
}

\author{
Debashis Ghosh $^{\dagger 1}$, Karen M. Haswell ${ }^{1}$, Molly Sprada ${ }^{2}$, Federico Gonzalez-Fernandez $z^{\dagger 2,3,4}$ \\ ${ }^{1}$ From the Department of Pharmacology, SUNY Upstate Medical University, Syracuse, New York \\ ${ }^{2}$ Medical Research Service of Veterans Affairs Medical Center, Buffalo, New York; \\ ${ }^{3}$ Ross Eye Institute, and Departments of Ophthalmology and Pathology \& Anatomic Sciences; ${ }^{4}$ SUNY \\ Eye Institute, State University of New York
}

Running Title: Is Zebrafish IRBP an enzyme?

Corresponding Authors:

Debashis Ghosh, Department of Pharmacology, SUNY Upstate Medical University, Room 6310, Weiskotten Hall, 750 East Adams St., Syracuse, NY 13210. Email: ghoshd@upstate.edu, Telephone: (315) 464-9677, Fax: (315) 464-8014

Federico Gonzalez-Fernandez, Medical Research Service, Veterans Affairs Medical Center, Building 20, Room 219, 3495 Bailey Ave., Buffalo, NY 14215. Email: fg23@buffalo.edu, Telephone: (716) 8632291, Fax: (858) 784-2779

Key words: Interphotoreceptor retinoid-binding protein, Interphotoreceptor matrix, retina, Visual cycle, Zebrafish, Oleic acid, X-ray structure

The abbreviations used are: IRBP, Interphotoreceptor retinoid-binding protein; zIRBP: Zebrafish IRBP; xIRBP, Xenopus IRBP; OLA, oleic acid; ATR, all-trans retinol; RPE, retinal-pigmented epithelium; IPM, interphotoreceptor matrix; CTPase, carboxy terminus processing protease. 


\begin{abstract}
Multiple functions for Interphotoreceptor Retinoid-Binding Protein (IRBP) may explain its localization in the retina, vitreous and pineal gland and association with retinitis pigmentosa and myopia. We have been engaged in uncovering the structure-function relationships of this interesting protein long thought to bind visual-cycle retinoids and fatty acids in the subretinal space. Although hydrophobic domains capable of binding such ligands have now been found, we ask what other structural domains might be present that could predict new functions? Interestingly, IRBP possesses a fold similar to C-terminal processing proteases (CTPases) but is missing the PDZ domain. Here we present structural evidence that this fold may have a role in a recently observed autoproteolytic activity of the two-module zebrafish (z) IRBP (Ghosh et al. Exp. Eye Res., 2015). When the structure of Scenedesmus obliquus D1 CTPase (D1P) is superimposed with the first module of zIRBP (z1), the PDZ domain of D1P occupies roughly the same position in the amino acid sequence as the inter-domain tether in $\mathrm{z} 1$, between residues P71 and P85. The catalytic triad K397, S372 and E375 of D1P is located at the inter-domain interfacial cleft, similarly as the tetrad K241, S243, D177 and T179 of z1 residues, presumed to have proteolytic function. Packing of two adjacent symmetry-related molecules within the zl crystal show that the helix $\alpha 8$ penetrates the interfacial cleft underneath the inter-domain tether, forming a simple intermolecular "knot". The fulllength zIRBP is cleaved at or immediately after T309, which is located at the end of $\alpha 8$ and is the ninth residue of the second module z2. We propose that the helix $\alpha 8$ within intact zIRBP bends at P301, away from the improbable knotted fold, and positions the cleavage site T309 near the putative catalytic tetrad of the neighboring zIRBP to be proteolytically cleaved. The conservation of this functional catalytic domain suggests that possible physiological roles of IRBP as a hydrolase needs to be considered.
\end{abstract}




\section{INTRODUCTION}

Once thought to simply bind visual-cycle retinoids in the subretinal space, Interphotoreceptor RetinoidBinding Protein (IRBP) appears to have multiple important roles outside vitamin A cycle (GonzalezFernandez, 2012). However, understanding these new roles requires defining its structure vis-a-vis function. IRBP is secreted by the rods and cones into the subretinal space where it is the major protein component of the interphotoreceptor matrix and interacts with domains of this scaffold particularly the cone matrix sheath(Garlipp and Gonzalez-Fernandez, 2013; Garlipp et al., 2011). IRBP has an unusual structure being composed of multiple modules (4 in tetrapods and human, and 2 to 3 modules in teleosts) (Nickerson et al., 2006; Rajendran et al., 1996) each composed of $\sim 300$ amino acid residues.

Early studies focused on the protein's role in retinoid trafficking between the photoreceptors, retinalpigmented epithelium (RPE) in the rod visual cycle, and more recently the cone visual cycle(Parker et al., 2009). Nevertheless, the exact role of IRBP in the visual cycle is not clear as its presence is not a strictly required under the experimental conditions examined (Ho et al., 1989; Palczewski et al., 1999). In any event, its role is clearly more than simply solubilizing the retinoids within the extracellular matrix as previously assumed. Finally, IRBP has free radical scavenging activities and can protect retinol from photodecomposition (Crouch et al., 1992; Gonzalez-Fernandez et al., 2015; Gonzalez-Fernandez et al., 2014; Parker et al., 2011). Mutations in IRBP have been implicated in autosomal recessive retinitis pigmentosa (den Hollander et al., 2009; Li et al., 2013).

Some important functions of IRBP may not involve the retinal visual cycle. For example, IRBP is present in the aqueous and vitreous, which is actually where it was first discovered. Although the function of IRBP in these compartments is unknown, reduced concentrations of IRBP in the aqueous and vitreous are associated with primary congenital glaucoma (Bouhenni et al., 2011) and diabetic retinopathy (Gao et al., 2008; Garcia-Ramirez et al., 2009), respectively. IRBP is also known to be expressed by the by the pineal gland. The function of IRBP in these extraretinal locations is unknown. Furthermore, IRBP may have a role in retinal development (Gonzalez-Fernandez and Healy, 1990; Gonzalez-Fernandez et al., 1993; Hessler et al., 1996; Liou et al., 1998) and myopia (Arno et al., 2015; Wisard et al., 2011).

The above functions are difficult to reconcile if IRBP is thought of only as a retinoid-binding protein in the subretinal space. Further clues to the function of IRBP may come from its structure. Although IRBP does contain hydrophobic domains capable of binding retinoids (Gonzalez-Fernandez et al., 2007; Gonzalez-Fernandez et al., 2009) and fatty acids (Ghosh et al., 2015), what other structural motifs are present that could contribute to the functions summarized above?

Interestingly, the only proteins known to share similarity with IRBP are members of two protein families not associated with vision, or with retinoid binding. From the agnatha to tetrapods, IRBP is composed of four homologous modules, each containing $\sim 300$ amino acid residues and two domains (Dettai and Lecointre, 2008). These domains have homology with C-terminal processing proteases (CTPase), and crotonases (Loew and Gonzalez-Fernandez, 2002). It was established early on that the tertiary structure of the second module of Xenopus IRBP had an overall fold similar to D1 C-terminal processing protease (D1P), but without the PDZ domain (Loew and Gonzalez-Fernandez, 2002). However, a potential enzymatic function has been ignored.

During a recent X-ray crystallographic study of oleic acid-binding to zebrafish IRBP (zIRBP) a possible autoproteolytic activity was observed (Ghosh et al., 2015). In that study, during formation of holo-zIRBP crystals the protein was cleaved near its center. A polypeptide containing the first module of zIRBP (z1) 
and only nine residues of the second (z2) crystalized. In the present study, we further examined this phenomenon by examining the structure of the cleaved complex and comparing the structure of $\mathrm{z} 1$ with that of CTPase. Our study indicates that a functional enzymatic site is retained in IRBP suggesting an overlooked enzymatic function deserving further investigation.

\section{EXPERIMENTAL PROCEDURESS}

\section{Expression and purification}

The expression and purification of the full length zIRBP is described in a recent publication from our laboratories(Ghosh et al., 2015). The cDNA for IRBP gene II, the product of which is two tandem homologous modules of about 300 amino acids, was obtained by screening a cDNA library (Rajendran et al., 1996). The full-length zIRBP cDNA was subcloned with the addition of the TEV protease recognition site immediately downstream (3') to factor Xa recognition site and immediately upstream (5') of the zIRBP gene in the pET-30 Xa/LIC vector. Expression of the protein in Escherichia coli BL21(DE3) and selenomethionine (Se-Met)-substituted zIRBP has been described (Ghosh et al., 2015).

zIRBP was extracted from the $E$. coli by microfluidization in the presence of broad spectrum protease inhibitors (Sigma) and purified using an Äkta FPLC (GE Healthcare) at $4^{\circ} \mathrm{C}$. Briefly, the recombinant protein was captured on a $5 \mathrm{~mL}$ HisTrap HP column (GE Healthcare) and eluted on a imidazole gradient. TEV protease was then added and the cleaved zIRBP located in the unbound fractions of a second $5 \mathrm{~mL}$ HisTrap HP column. The cleaved zIRBP was further purified by charged anion exchange column (HiTrap Q HP, GE Healthcare) and eluted in a linear $\mathrm{NaCl}$ gradient. Fractions containing zIRBP were pooled and the $\mathrm{NaCl}$ concentration was adjusted to $100 \mathrm{mM}$. The purity of zIRBP was approximately $>98 \%$ by SDS-PAGE. The protein was then concentrated to $15 \mathrm{mg} / \mathrm{mL}$ and incubated with $439 \mathrm{M}$ oleic acid and for $90 \mathrm{~min}$. The solution was then filtered to remove particulate matter prior to crystallization.

\section{Crystallization, X-ray diffraction and structure determination}

The recombinant zIRBP was crystallized by the sitting-drop vapor diffusion at $23^{\circ} \mathrm{C}($ Ghosh et al., 2015). Diffraction data on the crystals of zIRBP were collected at the synchrotron X-ray beamline 19ID, the Structural Biology Center, Advanced Photon Source, Argonne National Laboratory, Argonne, IL. The structure was determined by a combination of MAD and molecular replacement methods (Ghosh et al., 2015).

As described in our previous study, (Ghosh et al., 2015) while elucidating the structure, we discovered that the two-module containing zIRBP polypeptide was proteolytically cleaved between the modules during crystallization, and only module 1 (309 amino acids of a total 594) crystallized. Most of the residues had well-defined electron densities for the side chains, which conformed to the known sequence. The final model included 309 amino acids, one oleic acid, three glycerol molecules, one HEPES and 166 water molecules. Coordinates and data are deposited in the Protein Data Bank under the accession code 4LUR.

\section{Sequence and 3D structure alignment}

The sequence alignments were performed using the Probabilistic Consistency-based Multiple Alignment of Amino Acid Sequences (ProbCons) (Do et al., 2005). The sequence and crystal structure of the zl was used as a template for the 3D structural alignment of the $z 2$. The MOE-Align and MOE-Homology platforms in the Molecular Operating Environment ${ }^{\circledR} 2012$ software (Chemical Computing Group, Montreal, Canada) were used for this purpose. MOE-Align generated a sequence alignment of z2 to the 
structure of $\mathrm{z} 1$ emphasizing the secondary structure similarity. MOE-Homology was used in generating the 3D structures of $z 2$ from the $z 1$ template. This method of alignment is based on a combination of the segment-matching procedure of Levitt (Levitt, 1992) and an approach to the modeling of indels based on that of Fechteler et al (Fechteler et al., 1995). Finally, the results were analyzed to determine the last residue of $\mathrm{z} 1$ and the beginning of the $\mathrm{z} 2$ module.

\section{RESULTS}

Sequence comparison of D1 C-terminal processing protease (D1P) and zIRBP module 1 (z1): Fig. 1 shows an alignment of the amino acid sequences of $z 1$ with D1P. The homologous sequences based on the homology of their 3-D structures are highlighted. The catalytic residues in D1P and those proposed for z1 are also indicated. Although sequence similarity is found between z1 and DIP, a region between z1 domains A and B is only present in DIP and not in $\mathrm{z} 1$. This sequence, which is highlighted yellow, is the DIP PDZ domain. Except for this domain, there is a significant homology between DIP and the N- and C-terminal z1 domains.

The full-length zIRBP (594 residues, theoretical $\mathrm{M}_{\mathrm{r}}=64,385$ daltons) has been successfully expressed and purified to homogeneity. On silver-stained SDS-PAGE, the purified zIRBP exhibits an estimated purity of greater than $95 \%$ and exhibits a single band at $\sim 67 \mathrm{kDa}$ corresponding to the full-length molecule even when concentrated to $5-20 \mathrm{mg} / \mathrm{ml}$ for crystallization (Fig. 2A). However, the concentrated protein displays gradual cleavage yielding an additional major band at about $37 \mathrm{kDa}$ (Fig. 2B), which grows several weeks at $23^{\circ} \mathrm{C}$.

X-ray diffraction data and analysis of crystals from different crystallization drops and purification experiments have established that only the polypeptide containing the first 309 residues crystallizes(Ghosh et al., 2015). This polypeptide was previously shown to contain 300 amino acids of module 1 (z1) and 9 of module 2 (z2). (We have used "module 1" or "z1" to denote synonymously, either the true repeating module sequence of the N-terminal 300 residues, or the crystallized cleavage product of 309 residues.) The presence beyond T309 of a few additional residues with high thermal motion and no discernable electron density cannot be ruled out. Nevertheless, no crystal of either the full length or the cleaved second module has yet been identified. The results indicate a time- and concentration-dependent cleavage of zIRBP into z1 with a homogeneity and mono-dispersity high enough to yield diffractionquality single crystals.

Retention of the C-terminal peptidase fold: hint of autocatalysis from crystal packing: An IRBP module has a fold similar to the MEROPS database (Rawlings et al., 2012) serine peptidase classification clan SK, family S41, subfamily A (CTPases), which includes CtpA, CtpB, and CtpC peptidases and chlamydial protease-like activity factor (CPAF) (Page and Di Cera, 2008; Rawlings et al., 2012). This subfamily typically recognizes certain sequences at the carboxyl terminus of proteins, and then processes C-terminal extensions and/or makes endoproteolytic cleavages at various distances from the C-terminal recognition site.

It was established early on that the tertiary structure of $\mathrm{x} 2 \mathrm{had}$ an overall fold similar to D1P without the PDZ domain (Loew and Gonzalez-Fernandez, 2002). Superposition of the structure of Scenedesmus obliquus D1P(Liao et al., 2000) with z1 (Fig. 3) as well as the sequence alignment in Fig. 1 clearly show that the N-terminal A domain of $\mathrm{z} 1$, and the C-terminal B domain in particular superimpose well with the corresponding domains of D1P. The intervening PDZ domain of D1P (Lad et al., 2007) is absent in z1 and replaced by the long and dynamically mobile extended chain between $\beta 0$ and $\alpha 4$, and residues P71 and P85, which acts as a tether between the A and B domains (Figs. 3, 4). There is no appreciable 
homology between the PDZ domain of D1P and the extended mobile chain ending in helix $\alpha 4$ (P71 to T96) in z1 (Fig. 4). Interestingly, the tether in $z 1$ is open wider than the same in $x 2$, thereby exposing the inter-domain interface, a four-stranded $\beta$-sheet formed by $\beta 4, \beta 5, \beta 8$, and $\beta 9$, to a greater extent.

Shown in Fig. 3, the catalytic residues K397, E375 and S372 of D1P (Inagaki et al., 2001; Liao et al., 2000) are situated between the analogous $\beta$-sheet and the B-domain at the interfacial cleft. A similar lysine K241 in z1 is situated only 3 residues upstream from K397 in D1P (Figs. 3, 4), and projects similarly into the interfacial cleft left wide open by the inter-domain tether. Furthermore, K241 is close proximity to the D177 and T179 side chains. These three side chains, together with one rotomer of the S243 side chain modeled in two conformations, constitute a tetrad of residues resembling the catalytic site of a protease/hydrolase (Hedstrom, 2002; Kraut, 1977; Page and Di Cera, 2008). The location and proximity of these residues suggest that any combination of them could potentially be involved in the hydrolysis of an ester bond.

Analysis of the packing of z1 molecules in the crystal reveals that the C-terminal helix of one molecule penetrates the opening underneath the inter-domain tether of the crystallographically-related neighboring molecule at the domain interface, forming what can be described as a simple intermolecular "knot" (Bolinger et al., 2010; Virnau et al., 2011; Yeates et al., 2007), as shown in Fig. 4A. Modeling of the electron density map and refinement of the model suggest that the polypeptide must have been cleaved at the $\mathrm{C}$ terminus of T309, or immediately thereafter, nine residues ${ }^{301}$ PALAQAAA ${ }^{309} \mathrm{~T}$ into the second module z2 of zIRBP. Alignment of the amino acid sequences of the zIRBP modules and homology modeling of the $z 2$ structure suggest this ${ }^{301}$ PALAQAAA ${ }^{309} \mathrm{~T}$ segment to be helical like $\alpha 1$ as the experimental structure data actually shows. However, since the extension of $\alpha 8$ via this segment and formation of the rest of the $\mathrm{z} 2$ structure through the inter-domain tether is topologically impossible, it is likely that the helix $\alpha 8$ for an intact full-length zIRBP bends at or around P301 and begins a new z1-like fold with the helix $\alpha 1$ for the second module, as indicated in Fig. 4B. Coincidentally, in this new orientation, the residues ${ }^{309}$ TLIAD of $\alpha 1$ (z2) are within the physical proximity to the putative catalytic residues D177, T179, K241 and S243 (Fig. 4B). Furthermore, when $\alpha 1$ of the homology-modeled z2 is superimposed with the new orientation of this $\alpha 8$ segment, $\mathrm{z} 2$ atoms remain clear of any major overlap or steric clash with those of $\mathrm{z} 1$ (result not shown), suggesting that the resulting tertiary fold of zIRBP is a distinct possibility. Upon cleavage at T309 or thereafter, the helix $\alpha 8$ releases the bending stress by straightening itself and forming the intertwined crystal packing interaction. This intricate intermolecular packing of z1 would have been unlikely if zIRBP molecules were cleaved by an exogenous protease prior to coming in close contacts with one another during nucleation.

\section{DISCUSSION}

An emerging concept is that IRBP has diverse roles including functions beyond the visual cycle.

Nevertheless, since its first descriptions more than three decades ago the literature has assumed that IRBP is simply an extracellular protein solubilizing retinoids within the interphotoreceptor matrix. We have been characterizing the structure of IRBP with the hope that such analysis will provide insight into the roles in the visual cycle as well as other functions.

Shortly after the sequence of IRBP became known, a similarity with a E. coli protease designated Tsp (tail-specific protease) was found (Silber et al., 1992a). Tsp is a member of a new family of proteases that proteolytically cleave hydrophobic C-termini. Although Tsp showed no significant homology to known protease sequences, a homology with IRBP was noted. Tsp does not bind retinol and recombinant module 1 of human IRBP does not show proteolytic activity against casein (Gross et al., 2000). 
These observations suggest that although a homology between IRBP and the CTPs exists, the two proteins do not share functions, suggesting that the sequence similarity is the result of maintenance of the same tertiary structure. An alternative explanation for IRBPs lack on enzymatic function is that the correct physiological substrate was not examined, multiple modules are needed for enzymatic function, and/or the IRBP needs to be modified to release its enzymatic function.

The possibility that IRBP could have an enzymatic function has not been further pursued. This may be because the conserved catalytic residues could not be identified in the primary sequence. However, the present structural analysis does demonstrate a tetrad of residues resembling the catalytic site of a protease/hydrolase (Hedstrom, 2002; Kraut, 1977; Page and Di Cera, 2008). It is not know if CTPs can cleave IRBP. The location and proximity of these residues suggest that any combination of them could potentially be involved in the hydrolysis of an ester bond. Interestingly, a proteolytic activity was previously associated with purified bovine IRBP (Plantner and Quinn, 1997). Although this activity was assumed to represent a contaminating metalloproteinase, a catalytic activity of IRBP could also have explained the data in that study.

The observation that the z1 molecules, when cleaved from the full-length zIRBP, pack with one other by forming an intermolecular "knot" with the free cleaved end is a clue to its possible autoproteolytic activity. Evidently, such a close molecule-to-molecule interaction and the resulting proteolysis would be more pronounced at higher protein concentrations, namely, during crystallization rather than during purification. Similarity of the folds, as well as the nature and locations of the putative catalytic residues of CTPases to those of $z 1$, suggest that auto-proteolysis is a distinct possibility. However, site-directed mutagenesis studies combined with crystallographic and enzymatic studies are necessary for an unequivocal answer to this question.

Previous studies did not recognize the potential catalytic site. Nevertheless, a similar lysine K241 in z1 is situated only 3 residues upstream from K397 in D1P, and projects similarly into the interfacial cleft left wide open by the inter-domain tether. Furthermore, K241 is close proximity to the D177 and T179 side chains. These three side chains, together with one rotomer of the S243 side chain modeled in two conformations, constitute a tetrad of residues resembling the catalytic site of a protease/hydrolase (Hedstrom, 2002; Kraut, 1977; Page and Di Cera, 2008). The location and proximity of these residues suggest that any combination of them could potentially be involved in the hydrolysis of an ester bond.

An unexpected finding, which supports an autocatalytic mechanism, was that the C-terminal helix of one molecule penetrates the opening underneath the inter-domain tether of its neighbor forming an intermolecular "knot" (Bolinger et al., 2010; Virnau et al., 2011; Yeates et al., 2007) (Fig. 4A). This suggests that cleavage occurred at or just beyond ${ }^{301}$ PALAQAAA ${ }^{309} \mathrm{~T}$ into $\mathrm{z} 2$. Interestingly the extension of this segment and formation of the rest of the $\mathrm{z} 2$ structure through the inter-domain tether is topologically impossible. As described in the Results, $\alpha 8$ bends at or around P301 and begins a new z1like fold with the helix $\alpha 1$ for the second module, as indicated in Fig. 4B. The remarkable finding is that the residues ${ }^{309}$ TLIAD of $\alpha 1(\mathrm{z} 2)$ are within the physical proximity to the putative catalytic residues D177, T179, K241 and S243 (Fig. 4B). Probably the cleavage at T309 or thereafter, the helix $\alpha 8$ releases the bending stress by straightening itself and forming the intertwined crystal packing interaction. This data supports an autocatalytic mechanism; the intricate intermolecular packing of $z 1$ would have been impossible if zIRBP molecules were cleaved by an exogenous protease.

Interestingly, for one member of the subfamily A of family S41, CPAF, activation is initiated by a concentration-dependent trans-autocatalytic cleavage that induces homo-dimerization and conformational changes that assemble the catalytic triad (Huang et al., 2008). This cleavage and assembly is followed by 
two more autocatalytic cleavages and removal of an inhibitor segment, which then allows for full activity. These cleavages occur in the middle of the CPAF zymogen (Huang et al., 2008). In addition to the classic $\mathrm{D} / \mathrm{E}-\mathrm{S}-\mathrm{H}$ triad, many peptidase/hydrolases have been shown to have catalytic residues such as K-S dyads, as well as S-E-D and S-H-H triads (Ekici et al., 2008; Page and Di Cera, 2008; Polgar, 2005). Threonine has also been implicated in the activation of S for the nucleophilic attack on the substrate (Bell et al., 2000). CTPases are generally not inhibited by serine protease inhibitors (Bowyer et al., 1992; Silber et al., 1992b; Yamamoto et al., 2001).

At present we cannot conclude whether this autocatalytic activity is only an in vitro artifact, or in fact is truly part of physiological important process. A challenge is that we do not known the identity of the native substrate. However, new developments in high throughput screening such as target-based highthroughput screening could provide a way to circumvent this problem (Niphakis and Cravatt, 2014; Schreiber et al., 2015). 


\section{REFERENCES}

Arno, G., Hull, S., Robson, A.G., Holder, G.E., Cheetham, M.E., Webster, A.R., Plagnol, V., Moore, A.T., 2015. Lack of Interphotoreceptor Retinoid Binding Protein Caused by Homozygous Mutation of RBP3 Is Associated With High Myopia and Retinal Dystrophy. Invest. Ophthalmol. Vis. Sci. 56, 23582365.

Bell, C.E., Frescura, P., Hochschild, A., Lewis, M., 2000. Crystal structure of the lambda repressor Cterminal domain provides a model for cooperative operator binding, Cell, pp. 801-811.

Bolinger, D., Sulkowska, J.I., Hsu, H.P., Mirny, L.A., Kardar, M., Onuchic, J.N., Virnau, P., 2010. A Stevedore's protein knot. PLoS Comput. Biol. 6, e1000731.

Bouhenni, R.A., Al Shahwan, S., Morales, J., Wakim, B.T., Chomyk, A.M., Alkuraya, F.S., Edward, D.P., 2011. Identification of differentially expressed proteins in the aqueous humor of primary congenital glaucoma. Exp. Eye Res. 92, 67-75.

Bowyer, J.R., Packer, J.C., McCormack, B.A., Whitelegge, J.P., Robinson, C., Taylor, M.A., 1992. Carboxyl-terminal processing of the D1 protein and photoactivation of water-splitting in photosystem II. Partial purification and characterization of the processing enzyme from Scenedesmus obliquus and Pisum sativum. J. Biol. Chem. 267, 5424-5433.

Crouch, R.K., Hazard, E.S., Lind, T., Wiggert, B., Chader, G., Corson, D.W., 1992. Interphotoreceptor retinoid-binding protein and alpha-tocopherol preserve the isomeric and oxidation state of retinol. Photochem. Photobiol. 56, 251-255.

den Hollander, A.I., McGee, T.L., Ziviello, C., Banfi, S., Dryja, T.P., Gonzalez-Fernandez, F., Ghosh, D., Berson, E.L., 2009. A homozygous missense mutation in the IRBP gene (RBP3) associated with autosomal recessive retinitis pigmentosa. Invest. Ophthalmol. Vis. Sci. 50, 1864-1872.

Dettai, A., Lecointre, G., 2008. New insights into the organization and evolution of vertebrate IRBP genes and utility of IRBP gene sequences for the phylogenetic study of the Acanthomorpha (Actinopterygii: Teleostei). Mol. Phylogenet. Evol. 48, 258-269.

Do, C.B., Mahabhashyam, M.S., Brudno, M., Batzoglou, S., 2005. ProbCons: Probabilistic consistencybased multiple sequence alignment. Genome Res. 15, 330-340.

Ekici, O.D., Paetzel, M., Dalbey, R.E., 2008. Unconventional serine proteases: variations on the catalytic Ser/His/Asp triad configuration. Protein Sci. 17, 2023-2037.

Fechteler, T., Dengler, U., Schomburg, D., 1995. Prediction of protein three-dimensional structures in insertion and deletion regions: a procedure for searching data bases of representative protein fragments using geometric scoring criteria. J. Mol. Biol. 253, 114-131.

Gao, B.B., Chen, X., Timothy, N., Aiello, L.P., Feener, E.P., 2008. Characterization of the vitreous proteome in diabetes without diabetic retinopathy and diabetes with proliferative diabetic retinopathy. $\mathrm{J}$ Proteome Res 7, 2516-2525.

Garcia-Ramirez, M., Hernandez, C., Villarroel, M., Canals, F., Alonso, M.A., Fortuny, R., Masmiquel, L., Navarro, A., Garcia-Arumi, J., Simo, R., 2009. Interphotoreceptor retinoid-binding protein (IRBP) is downregulated at early stages of diabetic retinopathy. Diabetologia.

Garlipp, M.A., Gonzalez-Fernandez, F., 2013. Cone outer segment and Muller microvilli pericellular matrices provide binding domains for interphotoreceptor retinoid-binding protein (IRBP). Exp. Eye Res. 113, 192-202.

Garlipp, M.A., Nowak, K.R., Gonzalez-Fernandez, F., 2011. Cone outer segment extracellular matrix as binding domain for interphotoreceptor retinoid-binding protein (IRBP). J. Comp. Neurol.

Ghosh, D., Haswell, K.M., Sprada, M., Gonzalez-Fernandez, F., 2015. Structure of Zebrafish IRBP Reveals Fatty Acid Binding. Exp. Eye Res.

Gonzalez-Fernandez, F., 2012. Interphotoreceptor retinoid binding protein; myths and mysteries. J Ophthalmic Vis Res 7, 100-104. 
Gonzalez-Fernandez, F., Baer, C.A., Ghosh, D., 2007. Module structure of interphotoreceptor retinoidbinding protein (IRBP) may provide bases for its complex role in the visual cycle - structure / function study of Xenopus IRBP. BMC Biochem 8, 15.

Gonzalez-Fernandez, F., Betts-Obregon, B., Yust, B., Mimun, J., Sung, D., Sardar, D., Tsin, A.T., 2015. Interphotoreceptor retinoid-binding protein protects retinoids from photodegradation. Photochem.

Photobiol. 91, 371-378.

Gonzalez-Fernandez, F., Bevilacqua, T., Lee, K.I., Chandrashekar, R., Hsu, L., Garlipp, M.A., Griswold, J.B., Crouch, R.K., Ghosh, D., 2009. Retinol-binding site in interphotoreceptor retinoid-binding protein (IRBP): a novel hydrophobic cavity. Invest. Ophthalmol. Vis. Sci. 50, 5577-5586.

Gonzalez-Fernandez, F., Healy, J.I., 1990. Early expression of the gene for interphotoreceptor retinolbinding protein during photoreceptor differentiation suggests a critical role for the interphotoreceptor matrix in retinal development. J. Cell Biol. 111, 2775-2784.

Gonzalez-Fernandez, F., Sung, D., Haswell, K.M., Tsin, A., Ghosh, D., 2014. Thiol-dependent antioxidant activity of interphotoreceptor retinoid-binding protein. Exp. Eye Res. 120, 167-174.

Gonzalez-Fernandez, F., Van Niel, E., Edmonds, C., Beaver, H., Nickerson, J.M., Garcia-Fernandez, J.M., Campohiaro, P.A., Foster, R.G., 1993. Differential expression of interphotoreceptor retinoid-binding protein, opsin, cellular retinaldehyde-binding protein, and basic fibroblastic growth factor. Exp. Eye Res. 56, 411-427.

Gross, E.A., Li, G.R., Lin, Z.Y., Ruuska, S.E., Boatright, J.H., Mian, I.S., Nickerson, J.M., 2000.

Prediction of structural and functional relationships of Repeat 1 of human interphotoreceptor retinoidbinding protein (IRBP) with other proteins. Mol. Vis. 6, 30-39.

Hedstrom, L., 2002. Serine protease mechanism and specificity. Chem. Rev. 102, 4501-4524.

Hessler, R.B., Baer, C.A., Bukelman, A., Kittredge, K.L., Gonzalez-Fernandez, F., 1996.

Interphotoreceptor retinoid-binding protein (IRBP): expression in the adult and developing Xenopus retina. J. Comp. Neurol. 367, 329-341.

Ho, M.T., Massey, J.B., Pownall, H.J., Anderson, R.E., Hollyfield, J.G., 1989. Mechanism of vitamin A movement between rod outer segments, interphotoreceptor retinoid-binding protein, and liposomes.

J.Biol.Chem. 264, 928-935.

Huang, Z., Feng, Y., Chen, D., Wu, X., Huang, S., Wang, X., Xiao, X., Li, W., Huang, N., Gu, L., Zhong, G., Chai, J., 2008. Structural basis for activation and inhibition of the secreted chlamydia protease CPAF. Cell Host Microbe 4, 529-542.

Inagaki, N., Maitra, R., Satoh, K., Pakrasi, H.B., 2001. Amino acid residues that are critical for in vivo catalytic activity of CtpA, the carboxyl-terminal processing protease for the D1 protein of photosystem II. J. Biol. Chem. 276, 30099-30105.

Kraut, J., 1977. Serine proteases: structure and mechanism of catalysis. Annu. Rev. Biochem. 46, 331358.

Lad, S.P., Yang, G., Scott, D.A., Wang, G., Nair, P., Mathison, J., Reddy, V.S., Li, E., 2007. Chlamydial CT441 is a PDZ domain-containing tail-specific protease that interferes with the NF-kappaB pathway of immune response. J. Bacteriol. 189, 6619-6625.

Levitt, M., 1992. Accurate modeling of protein conformation by automatic segment matching. J. Mol. Biol. 226, 507-533.

Li, S., Yang, Z., Hu, J., Gordon, W.C., Bazan, N.G., Haas, A.L., Bok, D., Jin, M., 2013. Secretory defect and cytotoxicity: the potential disease mechanisms for the retinitis pigmentosa (RP)-associated interphotoreceptor retinoid-binding protein (IRBP). J. Biol. Chem. 288, 11395-11406.

Liao, D.I., Qian, J., Chisholm, D.A., Jordan, D.B., Diner, B.A., 2000. Crystal structures of the photosystem II D1 C-terminal processing protease. Nat. Struct. Biol. 7, 749-753.

Liou, G.I., Fei, Y., Peachey, N.S., Matragoon, S., Wei, S., Blaner, W.S., Wang, Y., Liu, C., Gottesman, M.E., Ripps, H., 1998. Early onset photoreceptor abnormalities induced by targeted disruption of the interphotoreceptor retinoid-binding protein gene. J.Neurosci. 18, 4511-4520. 
Loew, A., Gonzalez-Fernandez, F., 2002. Crystal structure of the functional unit of interphotoreceptor retinoid binding protein. Structure 10, 43-49.

Nickerson, J.M., Frey, R.A., Ciavatta, V.T., Stenkamp, D.L., 2006. Interphotoreceptor retinoid-binding protein gene structure in tetrapods and teleost fish. Mol. Vis. 12, 1565-1585.

Niphakis, M.J., Cravatt, B.F., 2014. Enzyme inhibitor discovery by activity-based protein profiling. Annu. Rev. Biochem. 83, 341-377.

Page, M.J., Di Cera, E., 2008. Serine peptidases: classification, structure and function. Cell. Mol. Life Sci. $65,1220-1236$.

Palczewski, K., Van Hooser, J.P., Garwin, G.G., Chen, J., Liou, G.I., Saari, J.C., 1999. Kinetics of visual pigment regeneration in excised mouse eyes and in mice with a targeted disruption of the gene encoding interphotoreceptor retinoid-binding protein or arrestin. Biochemistry. 38, 12012-12019.

Parker, R., Wang, J.S., Kefalov, V.J., Crouch, R.K., 2011. Interphotoreceptor Retinoid-Binding Protein as the Physiologically Relevant Carrier of 11-cis-Retinol in the Cone Visual Cycle. J. Neurosci. 31, 47144719.

Parker, R.O., Fan, J., Nickerson, J.M., Liou, G.I., Crouch, R.K., 2009. Normal cone function requires the interphotoreceptor retinoid binding protein. J. Neurosci. 29, 4616-4621.

Plantner, J.J., Quinn, T.A., 1997. Association of matrix metalloproteinases with interphotoreceptor retinoid binding protein. Curr. Eye Res. 16, 51-55.

Polgar, L., 2005. The catalytic triad of serine peptidases. Cell. Mol. Life Sci. 62, 2161-2172.

Rajendran, R.R., Van Niel, E.E., Stenkamp, D.L., Cunningham, L.L., Raymond, P.A., GonzalezFernandez, F., 1996. Zebrafish interphotoreceptor retinoid-binding protein: differential circadian expression among cone subtypes. J. Exp. Biol. 199, 2775-2787.

Rawlings, N.D., Barrett, A.J., Bateman, A., 2012. MEROPS: the database of proteolytic enzymes, their substrates and inhibitors. Nucleic Acids Res. 40, D343-350.

Schreiber, S.L., Kotz, J.D., Li, M., Aube, J., Austin, C.P., Reed, J.C., Rosen, H., White, E.L., Sklar, L.A., Lindsley, C.W., Alexander, B.R., Bittker, J.A., Clemons, P.A., de Souza, A., Foley, M.A., Palmer, M., Shamji, A.F., Wawer, M.J., McManus, O., Wu, M., Zou, B., Yu, H., Golden, J.E., Schoenen, F.J., Simeonov, A., Jadhav, A., Jackson, M.R., Pinkerton, A.B., Chung, T.D., Griffin, P.R., Cravatt, B.F., Hodder, P.S., Roush, W.R., Roberts, E., Chung, D.H., Jonsson, C.B., Noah, J.W., Severson, W.E., Ananthan, S., Edwards, B., Oprea, T.I., Conn, P.J., Hopkins, C.R., Wood, M.R., Stauffer, S.R., Emmitte, K.A., 2015. Advancing Biological Understanding and Therapeutics Discovery with Small-Molecule Probes. Cell 161, 1252-1265.

Silber, K.R., Keiler, K.C., Sauer, R.T., 1992a. Tsp: a tail-specific protease that selectively degrades proteins with nonpolar C termini. 89, 295-299.

Silber, K.R., Keiler, K.C., Sauer, R.T., 1992b. Tsp: a tail-specific protease that selectively degrades proteins with nonpolar C termini. Proc. Natl. Acad. Sci. U. S. A. 89, 295-299.

Virnau, P., Mallam, A., Jackson, S., 2011. Structures and folding pathways of topologically knotted proteins. Journal of physics. Condensed matter : an Institute of Physics journal 23, 033101.

Wisard, J., Faulkner, A., Chrenek, M.A., Waxweiler, T., Waxweiler, W., Donmoyer, C., Liou, G.I., Craft, C.M., Schmid, G.F., Boatright, J.H., Pardue, M.T., Nickerson, J.M., 2011. Exaggerated eye growth in IRBP-deficient mice in early development. Invest. Ophthalmol. Vis. Sci. 52, 5804-5811.

Yamamoto, Y., Inagaki, N., Satoh, K., 2001. Overexpression and characterization of carboxyl-terminal processing protease for precursor D1 protein: regulation of enzyme-substrate interaction by molecular environments. J. Biol. Chem. 276, 7518-7525.

Yeates, T.O., Norcross, T.S., King, N.P., 2007. Knotted and topologically complex proteins as models for studying folding and stability. Curr. Opin. Chem. Biol. 11, 595-603. 


\section{ACKNOWLEDGEMENTS}

The work was supported by NIH R01 EY09412 (D.G., F.G.-F.), Merit Review Award I01BX007080 from the Biomedical Laboratory Research \& Development Service of the Veterans Affairs Office of Research and Development (F.G.-F.), R24 EY 016662 core instrumentation grant; and an Unrestricted Research Grant from Research to Prevent Blindness to the Department of Ophthalmology at State University of New York at Buffalo. The contents of this study do not represent the views of the Department of Veterans Affairs, or the United States Government. The Structural Biology Center at the Advanced Photon Source, Argonne, IL is operated by UChicago Argonne, LLC, for the U.S. Department of Energy, Office of Biological and Environmental Research under contract DE-AC02-06CH11357. 


\section{FIGURES}

\begin{tabular}{|c|c|c|c|c|c|c|c|}
\hline & & $\alpha$ & $\rightarrow$ & & $\longrightarrow$ & & $\longrightarrow$ \\
\hline $\mathrm{z} 1$ & 1 & FSPTLIADMA & KIFMDNYCSP & EKLTGMEEAI & DAASSNTEIL & SISDPTMLAN & VLTDGVKKTI \\
\hline \multirow[t]{2}{*}{ D1P } & 77 & -MVTSEQLLF & LEAWRAVDRA & YVDKSFNGQS & WFKLRETYLK & KEPMDRRAQT & YDAIRKLLAV \\
\hline & & $\leftarrow \beta 0 \rightarrow$ & & & & & \\
\hline z 1 & 61 & SDSRVKVTYE & 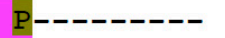 & ---------- & ---------- & ---------- & DLILAAPPAM \\
\hline D1P & 136 & LDDPFTRFLE & PSRLAALRRG & TAGSVTGVGL & EITYDGGSGK & DVVVLTPAPG & ---------- \\
\hline z 1 & 82 & PDIPLEHLAA & MIKGT----- & ---------- & -- & ---- & -0. \\
\hline \multirow[t]{2}{*}{ D1P } & 186 & --------- & $----G P A E K$ & AGARAGDVIV & TVDGTAVKGL & SLYDVSDLLQ & GEADSOVEVV \\
\hline & & & & $\leftarrow \beta 1 \rightarrow$ & & $\leftarrow$ & $\beta 2 \rightarrow$ \\
\hline z 1 & 97 & ---------- & ---------- & ----VKVEIL & $----E G N---$ & $---------I$ & GYLKIQHIIG \\
\hline \multirow[t]{2}{*}{ D1P } & 231 & LHAPGAPSNT & RTLQLTRQKV & TINPVTFTTC & SNVA---AAA & LPPGAAKQQL & GYVRLATTFN- \\
\hline & & & $-\alpha 5$ & & $\longrightarrow$ & $\leftarrow \beta 3 \rightarrow$ & $\leftarrow \alpha 6$ \\
\hline $\mathrm{z} 1$ & 117 & --EEM----- & $-------\mathrm{AQK}$ & VGPLLLEYIW & DKI---L--P & TSAMILDFRS & TVTGELSGIP \\
\hline \multirow[t]{2}{*}{$\mathrm{D} 1 \mathrm{P}$} & 287 & SN---TTAAA & QQAFTEL--- & ---------- & $---S K Q---G$ & VAGLVLDIRN & NGGGLFPAGV \\
\hline & & & $\leftarrow \beta 4 \rightarrow$ & $\leftarrow \beta 5$ & $\rightarrow$ & $\leftarrow$ & $\beta 6 \rightarrow$ \\
\hline $\mathrm{z} 1$ & 158 & YIVSYFTDPE & PLIHIDSVYD & RTAD-LTIEL & WSMPTLLGK- & --RYGTSKPL & IILTSKDTLG \\
\hline \multirow[t]{2}{*}{ D1P } & 325 & NVARMLVD-- & -RGDLVLIAD & SQGIR--DIY & SADGN----S & ID---SATPL & VVLVNRGTAS \\
\hline & & $-\alpha 7 \longrightarrow$ & $\leftarrow \beta 7 \rightarrow$ & $\leftarrow \beta$ & $\rightarrow$ & $\beta 9 \longrightarrow$ & \\
\hline $\mathrm{z} 1$ & 214 & IAEDVAYCLK & NLKRATIVGE & NTAGGTVKMS & KMKVG & VTVPVAKSIN & PITGKSWEIN \\
\hline \multirow[t]{2}{*}{$\mathrm{D} 1 \mathrm{P}$} & 373 & ASEVLAGALK & DSKRGLIAGE & RTFGKGLIQT & VVDLSDGSGV & AVTVARYQ-- & TPAGVDINKI \\
\hline & & & & $\alpha 8$ & $\longrightarrow \leftarrow \mathrm{z} 2 \mathrm{c}$ & $\rightarrow$ & \\
\hline z 1 & 274 & GVAPDVDVA- & $------A E D A$ & LDAAIAI IKL & RAEIPALAQA & AAT------- & -- \\
\hline $\mathrm{D} 1 \mathrm{P}$ & 431 & GVSPDVQLDP & EVLPTD---- & LEGVCRVLG- & --------- & ---SDAAPRL & $\mathrm{FG}$ \\
\hline
\end{tabular}

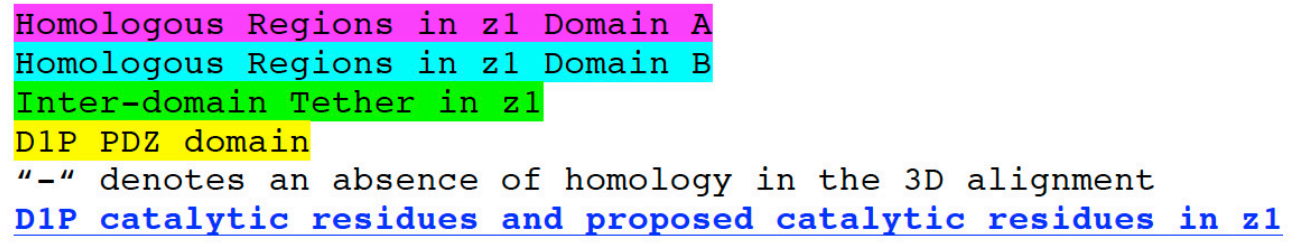

Figure 1. Alignment of the amino acid sequences of $\mathrm{z} 1$ and D1P. The homologous sequences based on the homology of their 3-D structures are highlighted. The catalytic residues in D1P and those proposed for z1 are also indicated. 


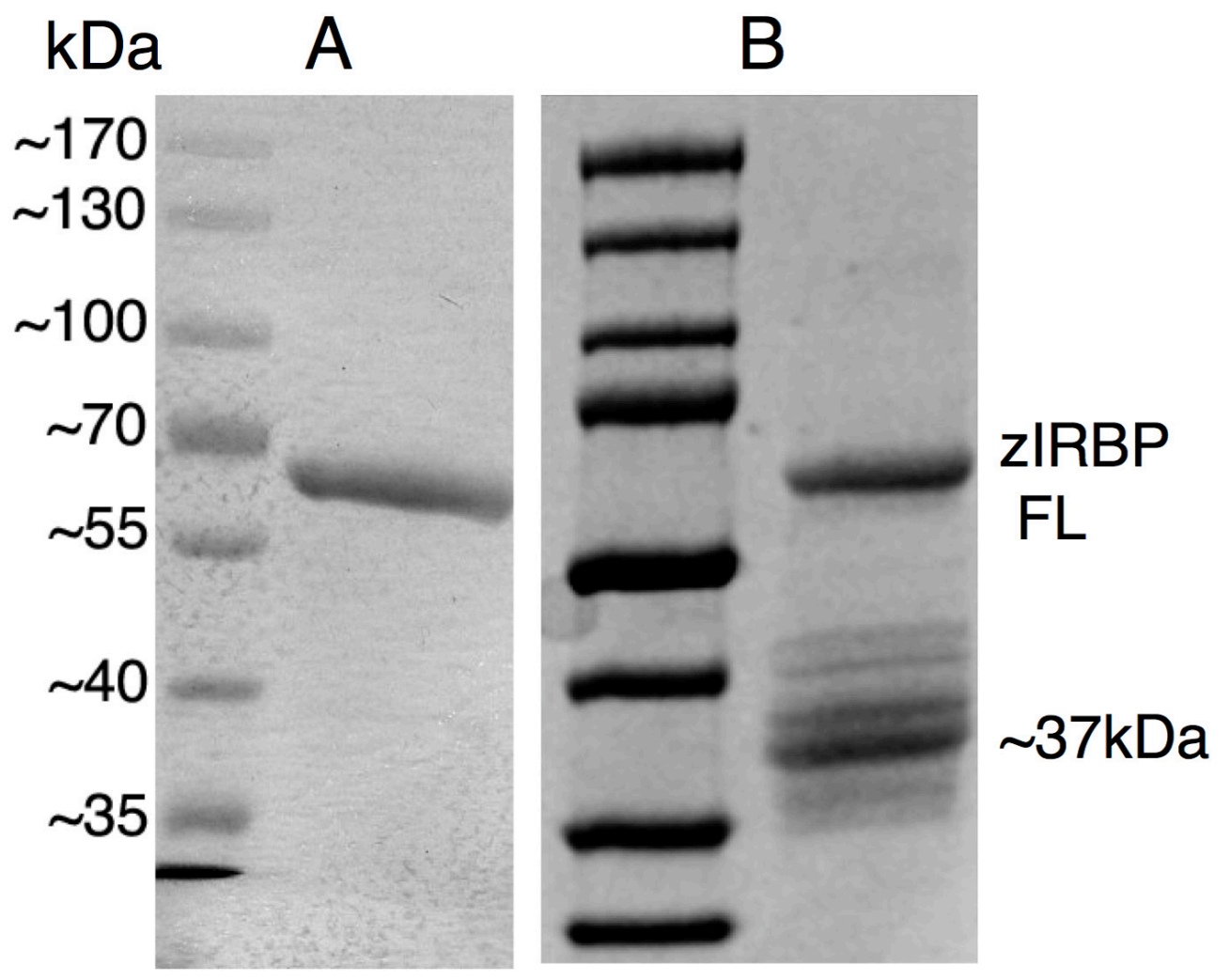

Figure 2. Proteolysis of purified zIRBP. (A) SDS-PAGE of purified full-length (FL) zIRBP as a single $\sim 67 \mathrm{kDa}$ band at a concentration of $20 \mathrm{mg} / \mathrm{ml}$, (B) proteolysis of zIRBP FL, analyzed after 2 weeks at room (crystallization) temperature, to primarily a $\sim 37 \mathrm{kDa}$ module 1 ( $\mathrm{z} 1$ ), which yields crystals. 


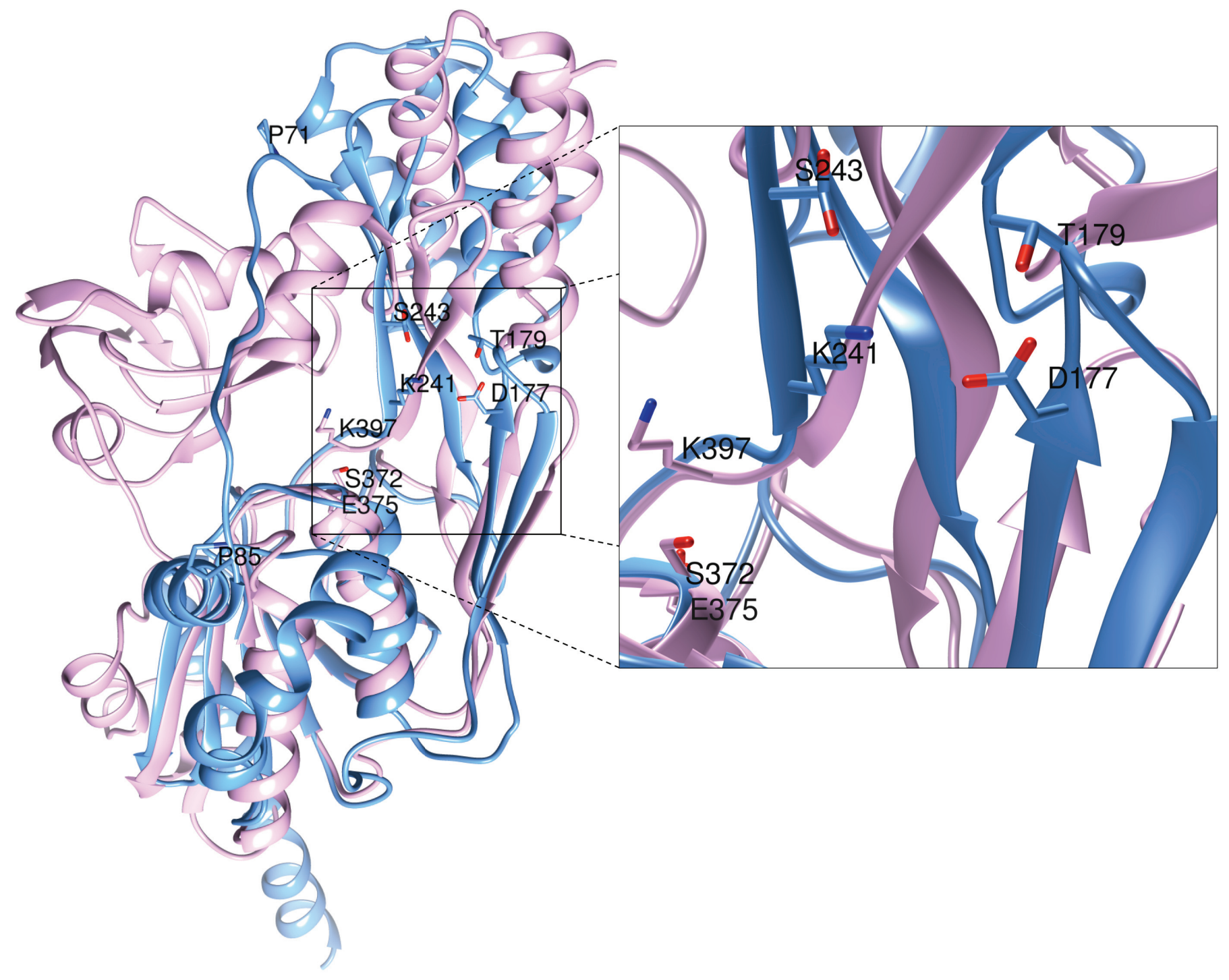

Figure 3. Superposition of the crystal structures of D1P (pink) and z1 (blue). Both A and B domains of the two structures overlay nicely. The PDZ domain of D1P, absent in zIRBP, occupies roughly the same position in the amino acid sequence as the inter-domain tether in z1, between residues P71 and P85. However, these two regions have no sequence or structural homology. The proteolytic triad K397, S372 and E375 of D1P are located at the inter-domain interfacial cleft, similarly as the tetrad K241, S243, D177 and T179 of z1 residues, presumed to have proteolytic function. 

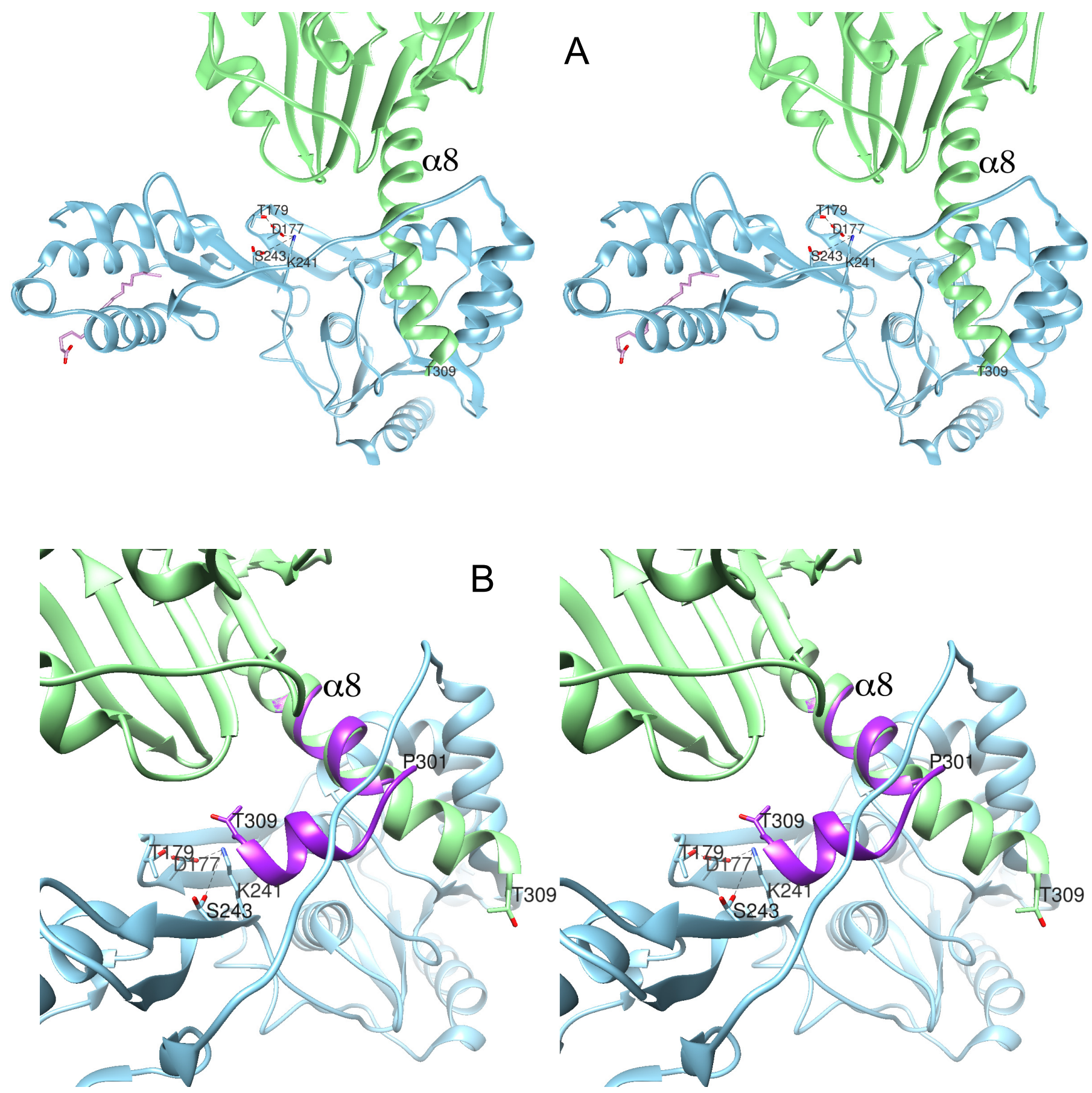

Figure 4. Stereodiagrams of intermolecular packing interactions within the $z 1$ crystal and clues to autoproteolysis. (A) Packing of two crystallographically related adjacent molecules within the z1 crystal. Helix $\alpha 8$ penetrates the inter-domain interfacial cleft underneath the inter-domain tether, forming a simple intermolecular "knot". The full-length zIRBP was cleaved at or immediately after the residue T309. (B) We postulate that the intact zIRBP would bend at or about P301, away from the improbable knotted fold as modeled in purple, thereby positioning the cleavage site near the putative catalytic tetrad. 